\title{
Destete y desempeño productivo de corderas en una pastura introducida en la Patagonia Austral
}

\section{Weaning and productive performance of lambs in an introduced pasture in South Patagonia}

Andrade M. ${ }^{1,2}$; Alvarez Bento ${ }^{3}$ J.; Utrilla V. ${ }^{1,2}$; Vargas P. ${ }^{1,2}$; Clifton G. ${ }^{1,2}$ y Gallardo R. ${ }^{2}$ andrade.miguel@inta.gob.ar; javieralvarezbento@gmail.com; utrilla.victor@inta.gob.ar

${ }^{1}$ Universidad Nacional de la Patagonia Austral. ICASUR. Departamento de Ciencias Exactas y Naturales. Unidad Académica Río Gallegos. Lisandro de La Torre 860. Río Gallegos. Santa Cruz. Argentina. Noviembre del 2018. Instituto Nacional de Tecnología Agropecuaria.

${ }^{3}$ Establecimiento Punta Loyola

Recibido: 26/11/2018. Aceptado: 03/04/2019

\section{RESUMEN}

En el SE de Santa Cruz, se desarrolló un trabajo con el objetivo de evaluar las pérdidas de corderas de reposición respecto al campo natural y la evolución del peso vivo desde enero a mayo durante dos años (2015-2016 y 2017-2018) en una pastura mezcla de pasto ovillo (Dactylis glomerata) y agropiro intermedio (Agropyron intermedium (Host) Beauvois. Tres lotes de corderas señaladas provenientes de tres campos fueron elegidas al azar y evaluadas. En los animales identificados se determinó en tres fechas (enero, febrero-marzo y abril-mayo) el peso vivo (PV), ganancias y rangos de PV. La pastura se muestreo en tres fechas (diciembre, marzo y mayo) para determinar la biomasa aérea disponible, el material vivo (MV) y muerto, la proteína bruta y digestibilidad de la materia seca. El análisis estadístico relaciono el PV de los animales con los días mediante regresiones y el PV de las corderas con el MV y las variables nutricionales mediante correlaciones. El trabajo incluyó registros de lluvias y temperaturas. En ambos años, se redujeron las pérdidas de animales en la pastura (< al 5\%) respecto al campo natural (18\%). La perfomance animal incluyó pérdidas de PV y mantenimiento del rango de PV inicial y final $\left(1^{\circ}\right.$ año) y mejoras en ambos parámetros $\left(2^{\circ}\right.$ año). Se concluye que el manejo aplicado genera un beneficio global y una mayor eficiencia en el sistema productivo aunque el desempeño animal depende del estado madurativo de la pastura influenciado por las condiciones hídricas ambientales.

Palabras Clave: Eficiencia del sistema; Forrajeras introducidas; Perfomance de ovinos jóvenes; Sur de Santa Cruz.

\begin{abstract}
We conducted an experiment in Southeast Santa Cruz, Patagonia, Argentina in order to evaluate losses of lambs respect to natural grassland, and evolution of live weight from January to May during two years (2015-2016 and 2017-2018) in a mixed pasture of orchardgrass (Dactylis glomerata) and intermediate wheatgrass (Agropyron intermedium (Host) Beauvois. Three flocks of marked ewes that came from three different paddocks were
\end{abstract}


chosen randomly and evaluated. Live weight (PV) and ranges of PV were determined on three dates (January, February-March-April-May). Pasture's available aerial biomass, the living (VM) and dead material, crude protein and dry matter digestibility were determined on three dates (December, March and May). Evolution of animal PV during the period was analyzed using regressions. Lamb PV was analyzed in relation to MV and nutritional variables through correlations. The work included records of rainfall and temperatures. Animal losses in the pasture were reduced $(<5 \%)$ respect to natural grassland $(18 \%)$ in both years. Animal performance included loss of PV and maintenance of range of initial and final PV (1st year) and improvements in both parameters (2nd year). We conclude that management of animals in pastures generates a global benefit and increased efficiency in the productive system although animal performance depends on the maturative state of the pasture, that are in turn influenced by water conditions of the environment.

Key words: Introduced forages; Perfomance of young ovines; South of Santa Cruz province; System efficiency.

\section{INTRODUCCIÓN}

En la Patagonia Austral, la productividad de los sistemas ganaderos es limitada y se sustenta en el aprovechamiento extensivo de los pastizales naturales, lo cual implica una alta variabilidad de la oferta forrajera debido a restricciones ambientales (fríos, nevadas y sequías) y al deterioro del pastizal por sobrepastoreo. En virtud de ello, se propone una mejora en los índices productivos de los sistemas ganaderos de la región en forma sustentable, a partir del uso estratégico de aquéllas áreas con mayor potencial (Proy. Reg. Territ. Santa Cruz, PRET SUR, 2013). Una alternativa válida para lograrlo consistiría en el pastoreo estratégico con ovinos de pasturas sembradas con gramíneas perennes introducidas en sitios favorables del sur de la región. Además, el pastoreo más controlado de estas forrajeras permitiría reducir las pérdidas de animales jóvenes respecto al manejo extensivo en campo natural (Andrade et al., 2017).

En este contexto, se desarrolló un trabajo con el objetivo de evaluar las pérdidas de corderas de reposición respecto al campo natural y la evolución del peso vivo desde enero a mayo durante dos años (2015-2016 y 2017-2018) en una pastura mezcla de pasto ovillo (Dactylis glomerata) y agropiro intermedio (Agropyron intermedium (Host) Beauvois en el SE de Santa Cruz.

\section{MARCO DE REFERENCIA}

En virtud de lo expuesto, existen antecedentes de pasturas instaladas en la Estepa Magallánica Húmeda (EMH) del SE de Santa Cruz con especies del género Agropyron (Utrilla et al., 2007), Dactylis y Festuca utilizadas con distintas categorías de ganado ovino (carneros, ovejas de refugo o descarte y/o corderos) para venta desde fines de la primavera y comienzo del verano hasta mediados de otoño, y con ovejas de cría y borregos desde ésta última época hasta principios de primavera (Informe Medio Término. PRET SUR, 2016. Citado por Andrade et al., 2017).

Asimismo, en la Estepa Magallánica Seca (EMS) del Sur de la provincia trabajos previos reportaron el desempeño productivo de ovinos y bovinos en pasturas sembradas de Agropiro intermedio (Agropyron intermedium (Host) Beauvois, Agropiro pubescente (Agropyron trichophorum (Link) Richter y mezcla de ambos. En estas pruebas, se reportaron ganancias de peso vivo y aumentos en la condición corporal, respectivamente, con borregas (Rivera et al., 
2012), ovejas de cría, borregas y corderas (Andrade et al., 2015), ovejas de refugo y corderas (Vargas et al., 2016; Utrilla et al., 2018) y novillitos de engorde (Clifton et al., 2012).

Además, con el propósito de complementar el uso estratégico con ganado ovino de pasturas instaladas, Alvarez Bento (com. pers.) informó una reducción en las pérdidas de corderas de reposición a partir del pastoreo más controlado de pasturas sembradas en la EMH respecto al manejo extensivo en campo natural (Citado por Andrade et al., 2017).

\section{MATERIALES Y MÉTODOS}

\section{Ubicación y descripción del sitio}

La experiencia se desarrolló en una pastura mezcla (550 has) de pasto ovillo (principal) y agropiro intermedio (acompañante) implantada en secano en el año 2013 en la Estancia Punta Loyola (distante $50 \mathrm{~km}$ al SE de Río Gallegos) ubicada en el área ecológica EMH del Sudeste de Santa Cruz (51 $\left.{ }^{\circ} 52^{\prime} 15,6^{\prime}{ }^{\prime} \mathrm{LS} 68^{\circ} 53^{\prime} 57,4^{\prime \prime} \mathrm{LO}\right)$. El clima del área es frío semiárido, con un régimen promedio anual de lluvias cercano a los $300 \mathrm{~mm}$ con algún máximo estival, y temperaturas medias anuales que oscilan entre 5,5 y 6,5 $\mathrm{C}$ (Oliva et al., 2001; Coronato et al., 2017). La vegetación natural del sitio son gramíneas (Festuca gracillima, principal, Poa spiciformis y Rytidosperma virescens, acompañantes) y graminoides (Carex spp.). El suelo de la pastura instalada presenta una textura Franco-Arenosa, $\mathrm{pH}$ medianamente ácido $(5,53)$, contenido de materia orgánica muy alto $(6,87 \%)$, valores de Nitrógeno total y Fósforo disponible, respectivamente, muy alto y bajo $(0,34 \%$ y $10,4 \mathrm{ppm})$ y sin problemas de salinidad (Fuente: Laboratorio de Suelos, EEA INTA Anguil, La Pampa).

\section{Mediciones climáticas}

En el establecimiento, se registraron las lluvias diarias con pluviómetro de campo (Datos Propios) y se procesaron, respectivamente, los valores mensuales de los años del trabajo y valores medios del período 2010-2014. Asimismo, se recolectaron las temperaturas máximas, medias y mínimas mensuales de Río Gallegos durante los años de la experiencia y se calculó la media de los valores del período 2011-2014 (Fuente: Sistemas de Información Patagonia Sur, SIPAS INTA. sipas.inta.gob.ar).

\section{Mediciones sobre la pastura}

En tres fechas de los años 2015-2016 (14/Dic, 01/Mar y 04/May) y 2017-2018 (22/Dic, 16/Mar y 14/May) se determinó la biomasa aérea disponible (BADisp) mediante el método Botanal (Tothill et al., 1992) y la composición botánica de la pastura (T'Mannetje y Haydock, 1963). Para ello, al inicio del trabajo y en sitios previamente establecidos se identificaron 5 muestras patrón de referencia. Luego, se realizó el muestreo en una transecta (dirección: SO - NE) mediante 50 estaciones de muestreo cada $50 \mathrm{~m}$, en las cuales se realizaron con un marco de $0,1 \mathrm{~m}^{2}$ estimaciones del patrón referente, registros de la cobertura aérea absoluta (\%) de la vegetación, mantillo o broza, suelo desnudo, pavimento de erosión y muerto en pie (Daubenmire, 1959) y se ranquearon las especies vegetales presentes. Además, a intervalos regulares se recolectaron 15 muestras referentes de forraje ( 5 patrones estimados $\mathrm{x} 3$ repeticiones) mediante cortes manuales de la vegetación con un marco de $0,1 \mathrm{~m}^{2}$. Finalmente, se midió con regla graduada la altura de tres plantas de pasto ovillo presentes por marco.

En gabinete, las muestras recolectadas se secaron en estufa a $60^{\circ} \mathrm{C}$ hasta peso constante para determinar la biomasa aérea disponible (BADisp) en $\mathrm{kg}$ MS/ha mediante la fórmula BADisp (g) x 100. Luego, se separaron 3 muestras representativas de forraje por fecha y año en Material Vivo (MV) y Muerto (MM) (conformados por hojas y tallos vivos y senescentes, respectivamente) para determinar la proporción relativa en base al material seco de ambas 
fracciones. Finalmente, se reconstituyeron las muestras originales del año 2015-2016 y se enviaron al laboratorio de la EEA INTA Manfredi (Córdoba) para su molienda en un molino Wiley® (Model 4 Mills, malla de $1 \mathrm{~mm}$ ) y determinación del contenido de Proteína Bruta $((\mathrm{PB}=$ Nitrógeno Total Kjeldahl $($ AOAC, 1995) x 6,25)) y la Digestibilidad In Vitro de la Materia Seca (DIVMS, Tilley y Terry, 1963).

\section{Registros de pérdidas de animales}

Se realizó el conteo inicial a la señalada (enero) y final (mayo) de las corderas que utilizaron la pastura en los años 2015-2016 ( $1^{\circ}$ año) y 2017-2018 ( $2^{\circ}$ año) y se determinó el número de animales faltantes entre ambas fechas.

\section{Elección y medición sobre los animales}

Se confeccionaron tres lotes de corderas de raza Corriedale elegidas al azar de animales señalados al momento de la esquila y provenientes de tres campos del establecimiento el $1^{\circ}$ (lotes: La Zanja, Argentino Abajo y Argentino Arriba) y $2^{\circ}$ año (lotes: Bajo Grande, La Zanja y Cerro Negro). El número total inicial de corderas evaluadas el $1^{\circ}$ y $2^{\circ}$ año, respectivamente, fue de 182 y 140, con rangos de animales por lote que oscilaron entre 29 y 82 y entre 36 y 63 animales, respectivamente. Previo al ingreso escalonado a la pastura en enero de ambos años, los animales de cada lote se identificaron con pintura y se determinó el peso vivo (PV) con balanza portátil en corrales anexos al galpón de esquila del establecimiento. Esta operación se repitió en febrero-abril y marzo-mayo del $1^{\circ}$ y $2^{\circ}$ año, respectivamente, en corrales móviles instalados en cercanías de la pastura.

\section{Procesamiento y análisis estadístico de los datos}

A partir del número de corderas faltantes al final del aprovechamiento de la pastura en los años evaluados, se determinó el porcentaje de pérdidas y se contrastó entre años y con valores previos en la pastura y en campo natural. Además, se realizaron cálculos del número de animales no faltantes a partir del uso inicial y sucesivo de la pastura y el beneficio de esta práctica de manejo en el establecimiento.

En ambos años, se realizaron determinaciones del porcentaje de corderas por rango de peso vivo (PV) y fecha para cada lote. Además, se calculó la media y el desvío estándar del PV por fecha y lote de animales y la ganancia de peso vivo (GPV) y diaria (GPVD) entre fechas y final. El análisis estadístico consistió en relacionar el PV de los animales por lote con los días transcurridos mediante regresiones polinomiales (PROC REG.SAS) y la media del PV de las corderas por lote con el MV $\left(1^{\circ}\right.$ y $2^{\circ}$ año $)$ y la DIVMS y PB $\left(1^{\circ}\right.$ año $)$ de la pastura mediante correlaciones (PROC CORR.SAS).

\section{RESULTADOS Y DISCUSIÓN}

\section{Variables Climáticas}

La pluviometría registrada en el lapso agosto-mayo del año 2015-2016 (84,1 $\mathrm{mm})$ se redujo $66 \%$ respecto al período 2010-2014 (255 mm) (Figura 1), lo cual representó un 34\% de lo esperable en condiciones normales y resultando en la temporada más seca de los últimos años según Andrade et al. (2017). Además, estos autores reportaron en los lapsos agosto-diciembre y enero-mayo del $1^{\circ}$ año valores pluviométricos que significaron 63 y $69 \%$ por debajo de la media del período informado. Por el contrario, en el lapso agosto-mayo del año 2017-2018 se registró un aumento del 30\% en las lluvias respecto al período 2011-2014 (331,7 mm versus $255 \mathrm{~mm}$ ), lo cual se manifestó principalmente en el intervalo enero-mayo (Figura 1). Finalmente, se reportó una diferencia apreciable $(247,6 \mathrm{~mm})$ a favor de las lluvias del $2^{\circ}$ año 
respecto al $1^{\circ}$ año del trabajo, lo cual implicó un aumento del 74,6 \% ocurrido principalmente en el lapso enero-mayo (Figura 1).

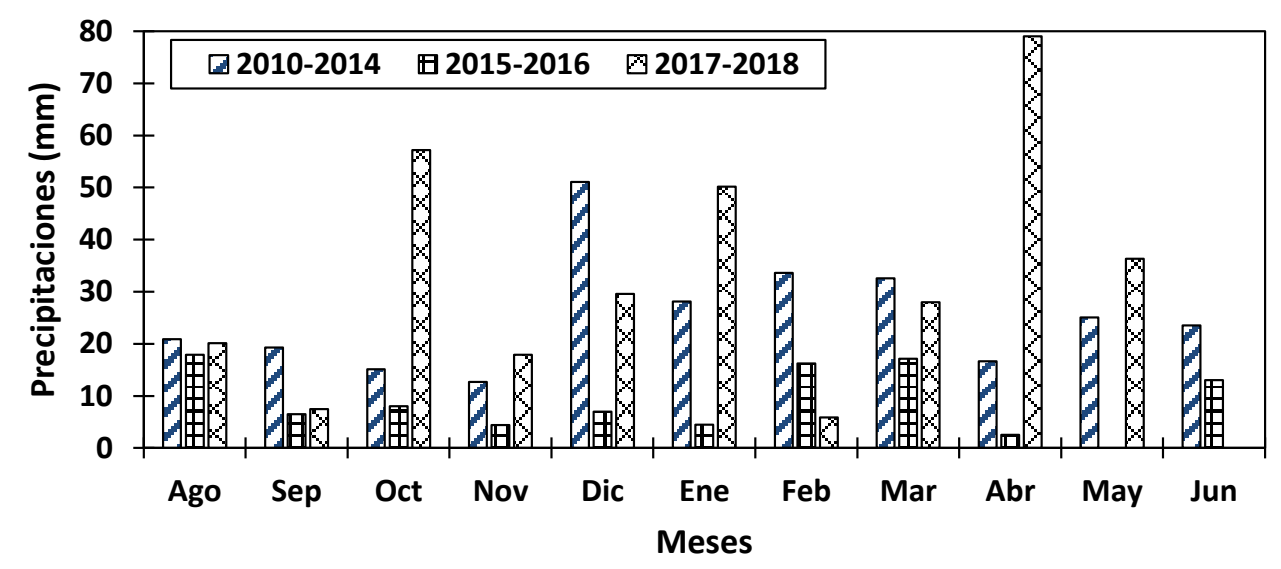

Figura 1: Distribución de las precipitaciones (mm) mensuales del período 2010-2014 y los años 2015-2016 y 2017-208. (Fuente: Ea. Punta Loyola).

En el lapso octubre-diciembre del período 2011-2014 y el año 2017-2018 hubo diferencias mínimas (hasta $0,5^{\circ} \mathrm{C}$ ) entre las temperaturas máximas, medias y mínimas, con valores que oscilaron, respectivamente, entre 13,6 y $18,1^{\circ} \mathrm{C}, 8,1$ y $11,7^{\circ} \mathrm{C}$ y 2,7 y $6,7^{\circ} \mathrm{C}$ (Figura $2 \mathrm{a}$, b y c). Con relación al lapso enero-marzo, las temperaturas máximas del $1^{\circ}\left(22,4\right.$ a $\left.19,6^{\circ} \mathrm{C}\right)$ y $2^{\circ}$ año $\left(22,5\right.$ a $\left.16,4^{\circ} \mathrm{C}\right)$ fueron mayores a los valores del período $2011-2014$ reportados $(17,3 \mathrm{a}$ $16,1^{\circ} \mathrm{C}$ ) con diferencias hasta $5^{\circ} \mathrm{C}$ (Figura 2a). Una respuesta similar para el mismo lapso registraron las temperaturas medias con valores para el $1^{\circ}$ y $2^{\circ}$ año y el período descripto, respectivamente, de 15,2 a $12,4^{\circ} \mathrm{C}, 14,1$ a $10,2^{\circ} \mathrm{C}$ y 12,7 a $10,6^{\circ} \mathrm{C}$ con diferencias hasta $4^{\circ} \mathrm{C}$ (Figura 2b). Además, las temperaturas mínimas del lapso enero-febrero del $1^{\circ}\left(7,9\right.$ y $\left.6,2^{\circ} \mathrm{C}\right)$ y $2^{\circ}$ año $\left(8,0\right.$ y $\left.7,6^{\circ} \mathrm{C}\right)$ superaron a los valores del período informado $\left(7,1\right.$ y $\left.5,2^{\circ} \mathrm{C}\right)$ con diferencias hasta $2,5^{\circ} \mathrm{C}$ (Figura 2c). Finalmente, las temperaturas máxima, media y mínima del $1^{\circ}$ año fueron mayores en enero y menores en febrero respecto al $2^{\circ}$ año (Figura $2 \mathrm{a}, \mathrm{b}$ y c). 


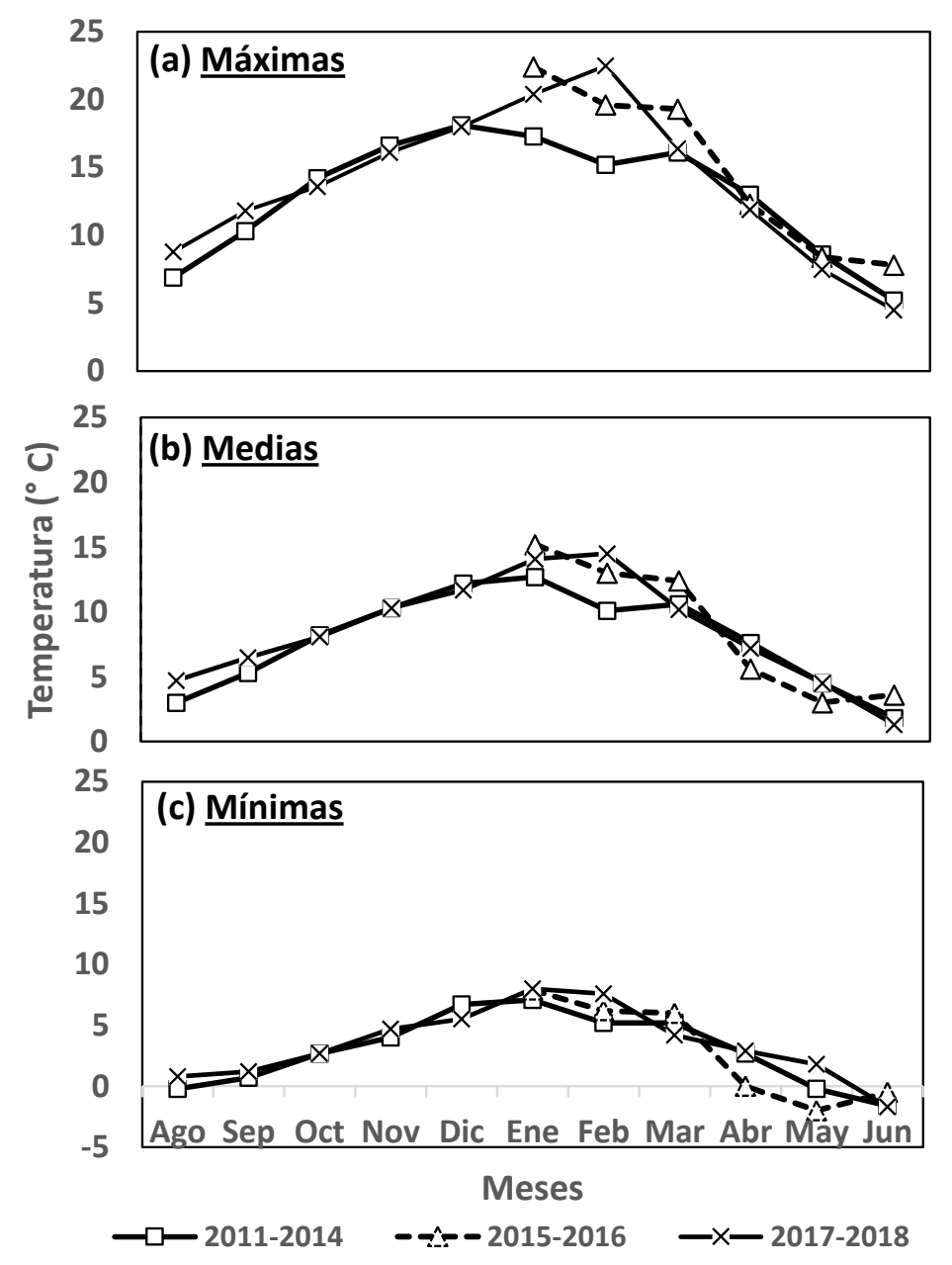

Figura 2: Valores de temperaturas máximas (a), medias (b) y mínimas (c) mensuales del período 2011-2014 y los años 2015-2016 y 2017-208. Lugar: Río Gallegos. (Fuente:

Sistemas de Información Patagonia Sur (SIPAS) INTA. sipas.inta.gob.ar)

\section{Caracterización de la pastura}

En ambos años, la cobertura vegetal de la pastura oscilo entre 24,5 y $35,5 \%$, con predominio del pasto ovillo (> al 90\%) respecto al agropiro intermedio (< al 10\%) (Tabla 1). El mantillo o broza relevado fue superior en las fechas inicial (diciembre) y final (mayo) del $1^{\circ}$ año $(40 \%)$ en relación a las mismas fechas del $2^{\circ}$ año (20 y 15\%) (Tabla 1 y Figura $3 a$ y b). Esta respuesta diferencial resultaría del material senescente acumulado proveniente del avance madurativo precoz de las plantas favorecido por las condiciones hídricas limitantes del $1^{\circ}$ año. Asimismo, la variación del mantillo estaría asociada con una cobertura del suelo desnudo menor en ambas fechas del $1^{\circ}$ año $\left(<\right.$ al 35\%) respecto al $2^{\circ}$ año $(>$ al 50\%) (Tabla 1$)$. Cabe mencionar que, Garzón (com. pers.) y de Caso (com. pers.) informaron en abril valores de cobertura vegetal, mantillo y suelo desnudo, respectivamente, cercanos al 45,35 y $25 \%$ en una pastura mezcla de pasto ovillo (predominante), festuca alta y agropiro alargado implantada en la EMH con 6-7 años de antigüedad y utilizada con ganado ovino. 
Tabla 1: Medias ( $\% \pm$ desvío estándar) de la cobertura del suelo y la composición botánica de la vegetación por fecha durante los años 2015-2016 y 2017-2018.

\begin{tabular}{|lccc|ccc|}
\hline Año/Fecha/ & \multicolumn{3}{c|}{ 2015-2016 } & \multicolumn{3}{c|}{ 2017-2018 } \\
\cline { 2 - 7 } Parámetro & 14/Dic & 01/Mar & 04/May & 22/Dic & 16/Mar & 14/May \\
\hline Cobertura del suelo & & & & & \\
Vegetación & $35,2 \pm 7,9$ & $35,5 \pm 17,3$ & $24,5 \pm 9,3$ & $24,5 \pm 7,0$ & $35,0 \pm 9,5$ & $34,0 \pm 13,4$ \\
Mantillo o broza & $39,4 \pm 10,3$ & $27,7 \pm 14,3$ & $37,9 \pm 14,0$ & $20,9 \pm 10,2$ & $26,6 \pm 16,1$ & $14,6 \pm 10,4$ \\
Suelo Desnudo & $25,3 \pm 11,4$ & $35,3 \pm 18,6$ & $33,6 \pm 17,5$ & $53,8 \pm 13,0$ & $37,4 \pm 17,1$ & $51,2 \pm 18,9$ \\
Pavimento de erosión & $0,1 \pm 0,7$ & $2,0 \pm 3,8$ & $0,1 \pm 0,7$ & & & \\
Muerto en pie & & & $3,7 \pm 4,2$ & $2,5 \pm 1,0$ & $3,4 \pm 2,7$ & $4,3 \pm 1,5$ \\
\hline Composición Botánica & & & & & & \\
Pasto ovillo & 90,8 & 97,9 & 96,3 & 94,6 & 95,9 & 84,4 \\
Agropiro intermedio & 8,9 & 2,0 & 3,7 & 5,4 & 4,1 & 5,2 \\
Otras especies & 0,4 & 0,1 & 0,0 & 0,0 & 0,0 & 10,4 \\
\hline
\end{tabular}
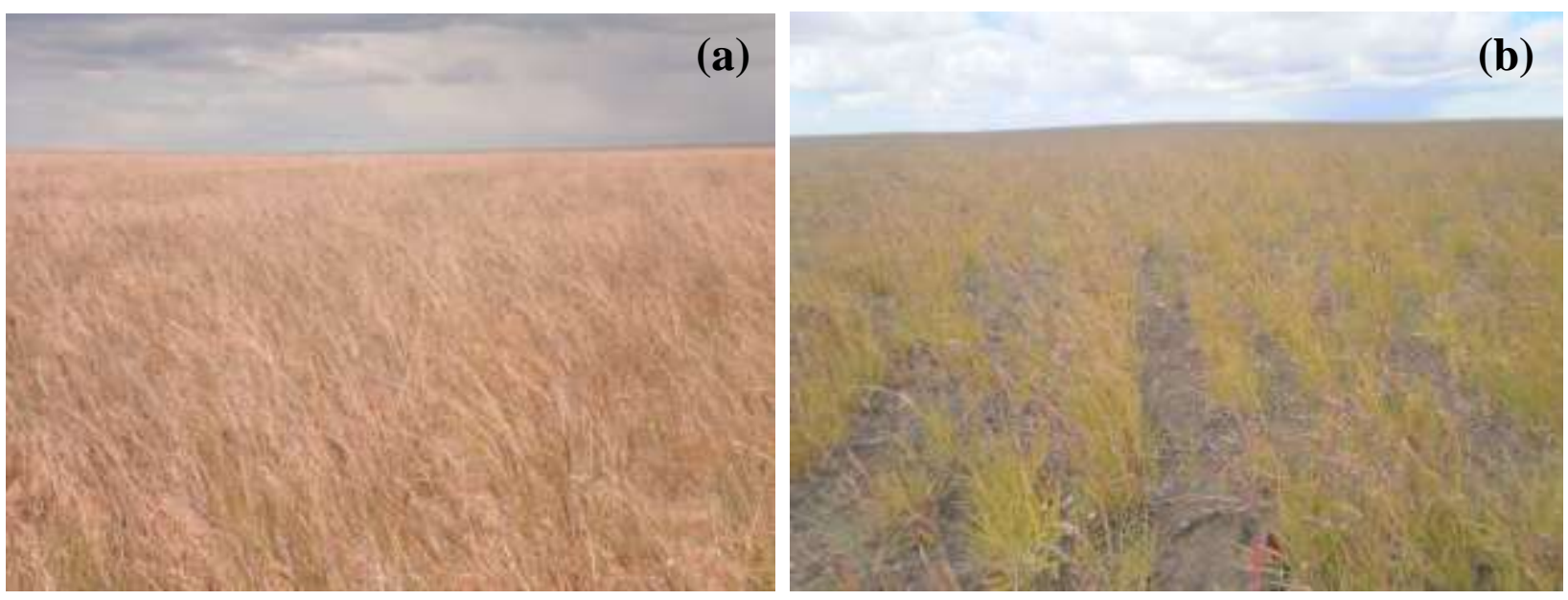

Figura 3: Vista inicial de la pastura en diciembre de los años 2015-2016 (a) 2017-2018 (b)

\section{Biomasa aérea disponible y altura de plantas}

En diciembre de ambos años las muestras patrón referentes más frecuentes de la pastura fueron 2,0 y 3,0 (Figuras 4 y 5 a y b). Asimismo, en marzo y mayo del $1^{\circ}$ año, se estimaron con mayor frecuencia los patrones 2,0 y 1,5. En cambio, en las mismas fechas del $2^{\circ}$ año los patrones estimados predominantes fueron, respectivamente, 3,0 y 2,0 y 2,0, 2,5 y 3,0 (Figura 4). En correspondencia con lo descripto, la BADisp de la pastura varió en el intervalo diciembre-mayo del $1^{\circ}$ y $2^{\circ}$ año, respectivamente, desde 1550 hasta $300 \mathrm{~kg} \mathrm{MS} / \mathrm{ha}$ y desde 1250 hasta $750 \mathrm{~kg} \mathrm{MS} / \mathrm{ha}$ (Figura 6). 


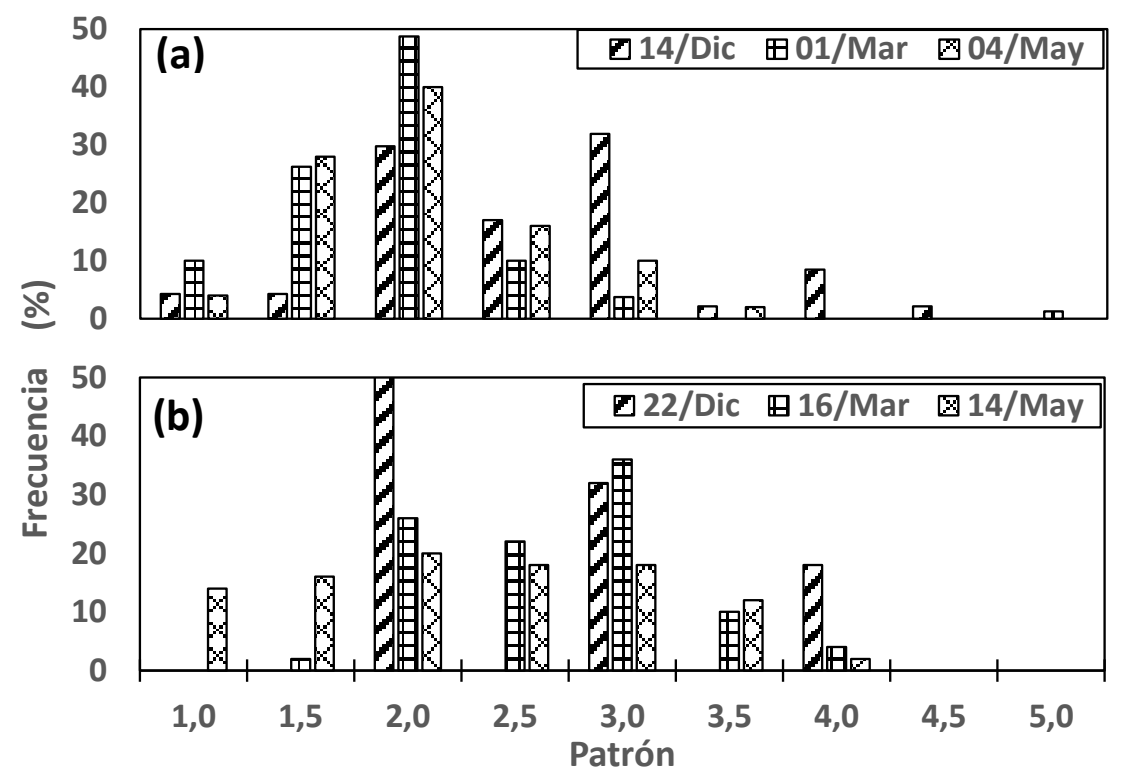

Figura 4: Frecuencia (\%) de los patrones estimados en la pastura por fecha de los años 20152016 (a) y 2017-2018 (b).
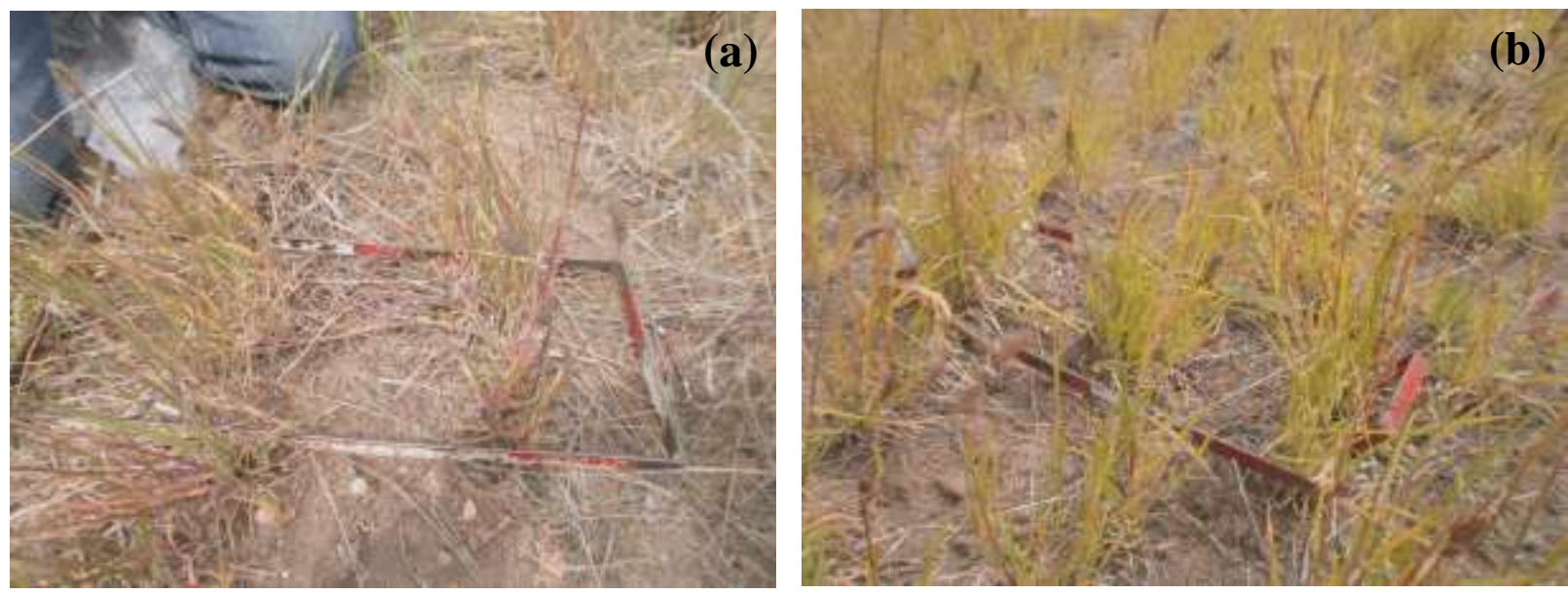

Figura 5: Vista de los patrones estimados más frecuentes en diciembre de los años 2015-2016 (a) y 2017-2018 (b).

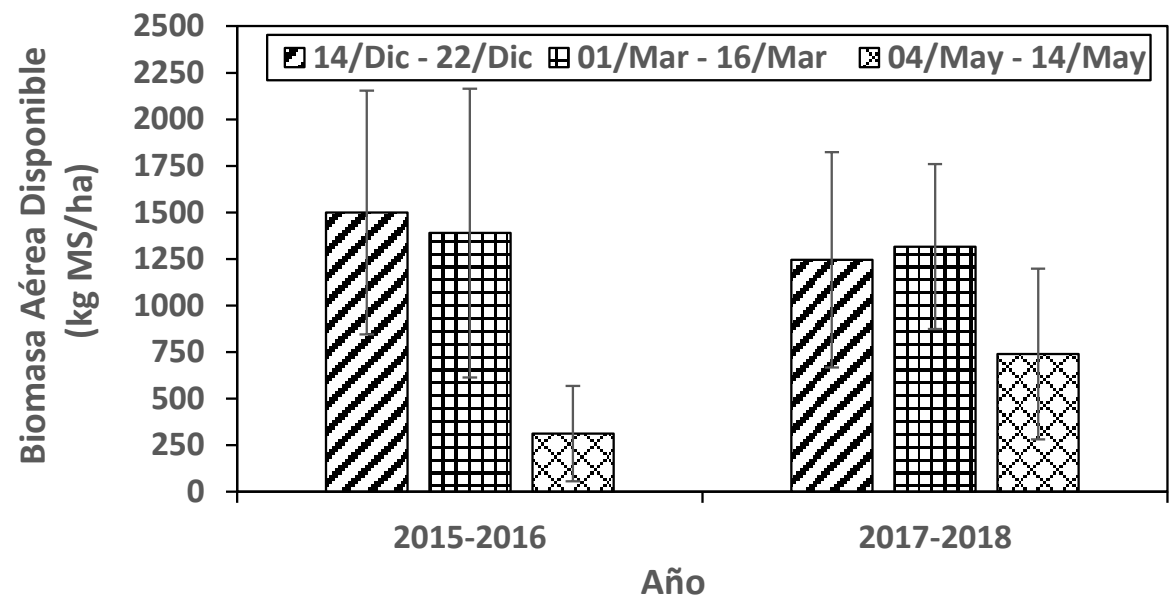

Figura 6: Medias de la biomasa aérea disponible (kg MS/ha \pm desvío estándar) de la pastura por fecha de los años 2015-2016 y 2017-2018. 
Cabe mencionar que, la biomasa reportada en diciembre de ambos años del trabajo fue mayor al forraje acumulado en la misma fecha en pasturas de agropiros crestado (Agropyron cristatum (L) Gaertn.), intermedio y pubescente (1000-1200 kg MS/ha) informados por Utrilla et al. (2007) y Utrilla et al. (2011) en la misma zona. Además, en concordancia con la BADisp final descripta en mayo del $1^{\circ}$ año, Garzón (com. pers.) informo un valor promedio remanente similar en abril en la pastura mezcla de pasto ovillo festuca alta y agropiro alargado implantada en la EMH y pastoreada con una alta carga de ganado ovino.

Asimismo, al comparar los valores inicial y final relevados en la pastura, la BADisp se redujo 5 y 1,5 veces el $1^{\circ}$ y $2^{\circ}$ año, respectivamente, en virtud del pastoreo recibido (carga animal inicial respectiva: 6,1 y 4,7 anim/ha) principalmente en el período marzo-mayo de ambos años. En cambio, la altura de plantas del $1^{\circ}$ y $2^{\circ}$ año varió, respectivamente, desde $14,1 \pm 4,4$ $17,8 \pm 4,8 \mathrm{~cm}$ (diciembre) hasta 12,2 $\pm 3,6-4,7 \pm 1,9 \mathrm{~cm}$ (mayo), con valores intermedios en marzo de 13,7 $\pm 6,0-11,6 \pm 4,3 \mathrm{~cm}$. En relación a ello, las alturas de pasto ovillo reportadas en diciembre fueron inferiores a los valores informados en agropiros por Utrilla et al. (2007) en la misma fecha y zona.

\section{Material vivo y muerto y calidad nutricional}

En diciembre del $2^{\circ}$ año, el forraje cosechado en la pastura estuvo representado principalmente por la fracción viva $(80 \%)$ (Figura 7) proveniente del material foliar verde recolectado del crecimiento favorecido por mejores condiciones hídricas (Figura 1) y térmicas previas (Figura 2). En concordancia con ello, en la misma fecha Utrilla et al. (2007) y Utrilla et al. (2017) informaron una ocupación del MV superior al 80 y $90 \%$ en pasturas de agropiro intermedio y pubescente de la EMH y EMS, respectivamente, lo cual indicaría un mayor crecimiento foliar de los agropiros. Cabe mencionar que, Andrade et al. (2017) reportaron en diciembre del $1^{\circ}$ año de la experiencia una menor ocupación (75\%) del MV cosechado en la pastura respecto al $2^{\circ}$ año, lo cual resultaría de una mayor senescencia foliar debido a las condiciones hídricas ambientales limitantes (Figura 1). En cambio, con el avance fenológico de las plantas la participación del MV en el $2^{\circ}$ año se redujo en forma considerable, lo cual implicó una ocupación apreciable del MM en marzo (80\%) y mayo (> al 90\%) (Figuras 7 y 8a) en virtud de la madurez avanzada de hojas y tallos del pasto ovillo. Una respuesta similar ocurrió el $1^{\circ}$ año (Figura 8b), aunque también favorecida por la menor pluviometría registrada (Figura 1). Por ello, el MM de la vegetación fue aún superior en marzo a los agropiros de la EMH descriptos con anterioridad (Utrilla et al., 2007).

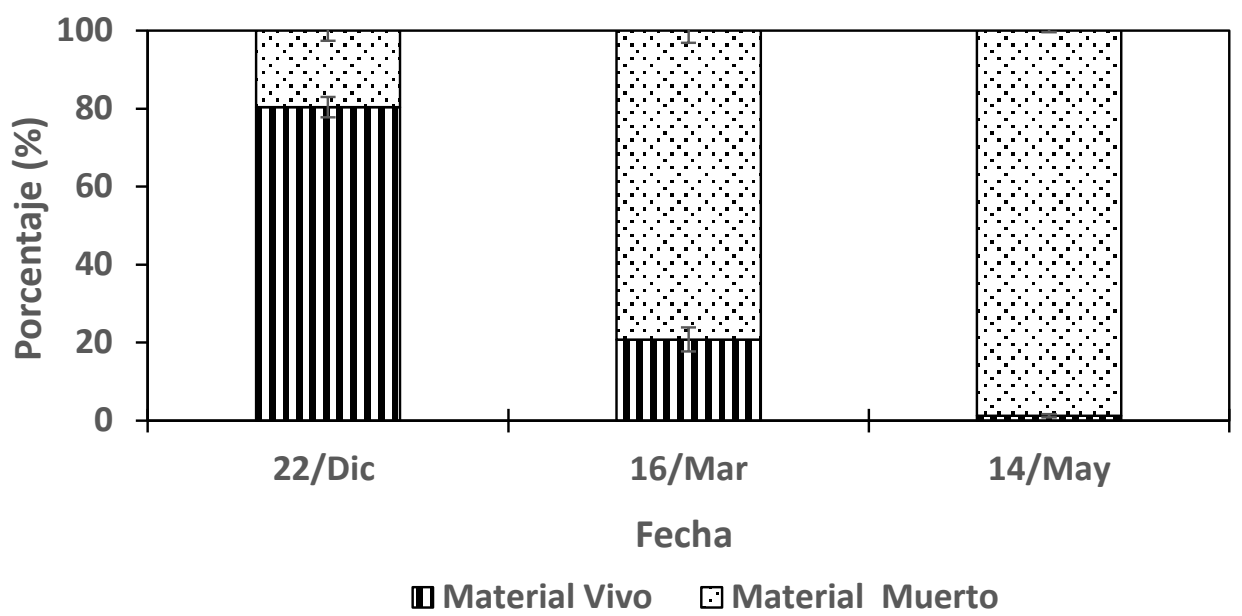

Figura 7: Porcentaje (\% \pm desvío estándar) del material vivo y muerto de la pastura por fecha en el año 2017-2018. 

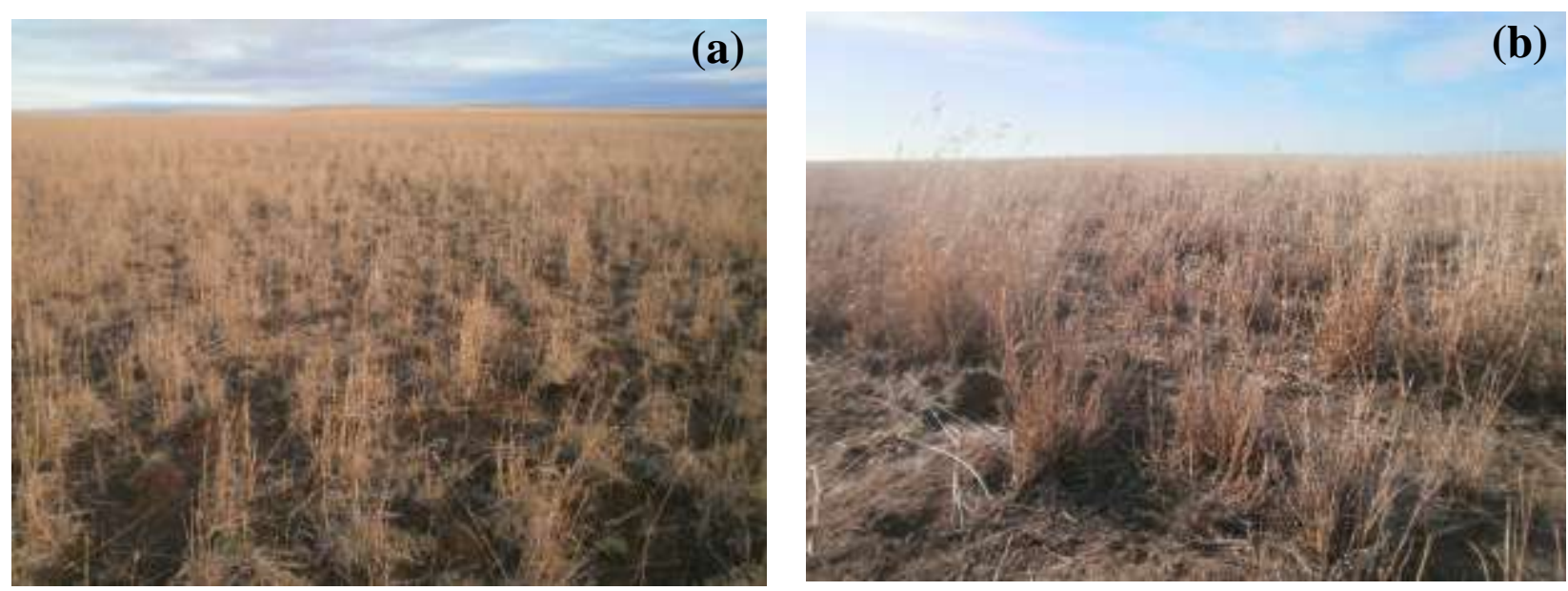

Figura 8: Vista final de la pastura en mayo de los años 2017-2018 (a) y 2015-2016 (b).

Asimismo, en diciembre del $1^{\circ}$ año Andrade et al. (2017) reportaron un contenido de PB y DIVMS del forraje cosechado inferiores al 5 y $70 \%$, respectivamente, lo cual podría explicarse por la madurez prematura del pasto ovillo en virtud de las restricciones hídricas relevadas durante el lapso agosto-diciembre (Figura 1). Por ello, los valores informados fueron inferiores a aquéllos reportados en la misma fecha por Utrilla et al. (2011) y Utrilla et al. (2017) en pasturas de agropiros intermedio y pubescente de la EMH y EMS que oscilaron entre 7,0 y $9,5 \%$ y entre 75,0 y 76,5\% para PB y DIVMS, respectivamente. Asimismo, el avance fenológico y el progreso de la estación de crecimiento del pasto ovillo generó reducciones en los parámetros descriptos, aunque principalmente en el contenido de PB de la vegetación (Andrade et al., 2017), lo cual se debería al aumento de las paredes celulares de los tejidos y la lignificación principal de los tallos conforme avanza la madurez de la gramínea (Holmes, 1980. Citado por Galli, 1997; Munro y Walters, 1985; Hodgson, 1990). En virtud de ello, los valores de PB y DIVMS reportados en marzo $(<$ al 3 y 55\%, respectivamente) por Andrade et al. (2017) fueron similares a aquellos informados por Utrilla et al. (2018) para la misma fecha en la pastura de agropiros de la EMS.

\section{Pérdidas de animales}

El manejo controlado de las corderas destetadas en la pastura durante el lapso enero-mayo de ambos años, logró reducir 5 ( $1^{\circ}$ año) y 11 veces $\left(2^{\circ}\right.$ año) la pérdida de animales respecto al destete tradicional de los animales en el campo natural (790 animales), lo cual significó disminuir el porcentaje de pérdidas a valores inferiores al 5\% en ambos casos comparado con el 17,9\% tradicional (Tabla 2). Además, el número de animales faltantes el año 2018 se redujo 2,5 veces respecto a las pérdidas relevadas el año 2016 (Tabla 2).

Tabla 2: Número de corderas en enero y mayo y porcentaje (\%) de animales faltantes según el manejo del destete por año.

\begin{tabular}{|c|c|c|c|c|c|}
\hline Año/ & 2014 & 2015 & 2016 & 2017 & 2018 \\
\hline $\begin{array}{l}\text { Destete/ } \\
\text { Fecha }\end{array}$ & $\begin{array}{c}\text { Campo Natural } \\
\text { (Tradicional) }\end{array}$ & $\begin{array}{c}\text { Campo Natural y } \\
\text { Pastura }\end{array}$ & Pastura & Pastura & Pastura \\
\hline Enero (Señalada) & 4403 & 4200 & 3355 & 1966 & 2600 \\
\hline Mayo & 3613 & 3818 & 3192 & 1856 & 2530 \\
\hline Diferencia faltante & 790 & 382 & 163 & 110 & 70 \\
\hline Porcentaje (\%) & 17,9 & 9,1 & 4,9 & 5,6 & 2,7 \\
\hline
\end{tabular}


Asimismo, esta práctica de manejo del destete de las corderas en la pastura permitió una mejora en el número de animales no faltantes analizados y por lo tanto un beneficio progresivo anual y total de este sistema utilizado en el establecimiento (Tabla 3).

Tabla 3: Número de corderas faltantes según el manejo de destete, número de animales no faltantes y beneficio (\%) del sistema utilizado por año y total.

\begin{tabular}{|lcccccc|}
\hline $\begin{array}{l}\text { Año/ } \\
\text { Pérdidas }\end{array}$ & 2014 & 2015 & 2016 & 2017 & 2018 & Total (4 años) \\
\hline $\begin{array}{l}\text { Manejo Tradicional } \\
\text { (se asume un valor histórico del 17,94\%) }\end{array}$ & 790 & 754 & 602 & 353 & 467 & 2176 \\
A Campo (según datos reales en función & 790 & 382 & 163 & 110 & 70 & 725 \\
(del manejo de destete) & & & & & & \\
\hline $\begin{array}{l}\text { Animales No Faltantes } \\
\text { Beneficio del Sistema Utilizado (\%) }\end{array}$ & 0 & 49,3 & 72,9 & 68,8 & 85,0 & 66,7 \\
\hline
\end{tabular}

\section{Perfomance animal}

En la fecha inicial del $1^{\circ}$ año (05-13/Ene), Andrade et al. (2017) informaron rangos de PV predominantes contrastantes entre los lotes de corderas evaluados (> 30 a $35 \mathrm{~kg}$ hasta $>20$ a $25 \mathrm{~kg}$ ). En cambio, en la misma fecha del $2^{\circ}$ año, las diferencias entre los rangos más frecuentes fueron menores con valores de PV incluidos en rangos $>30$ a $35 \mathrm{~kg}$ (Bajo Grande) hasta $>25$ a $30 \mathrm{~kg}$ (La Zanja y Cerro Negro) (Figura 9).
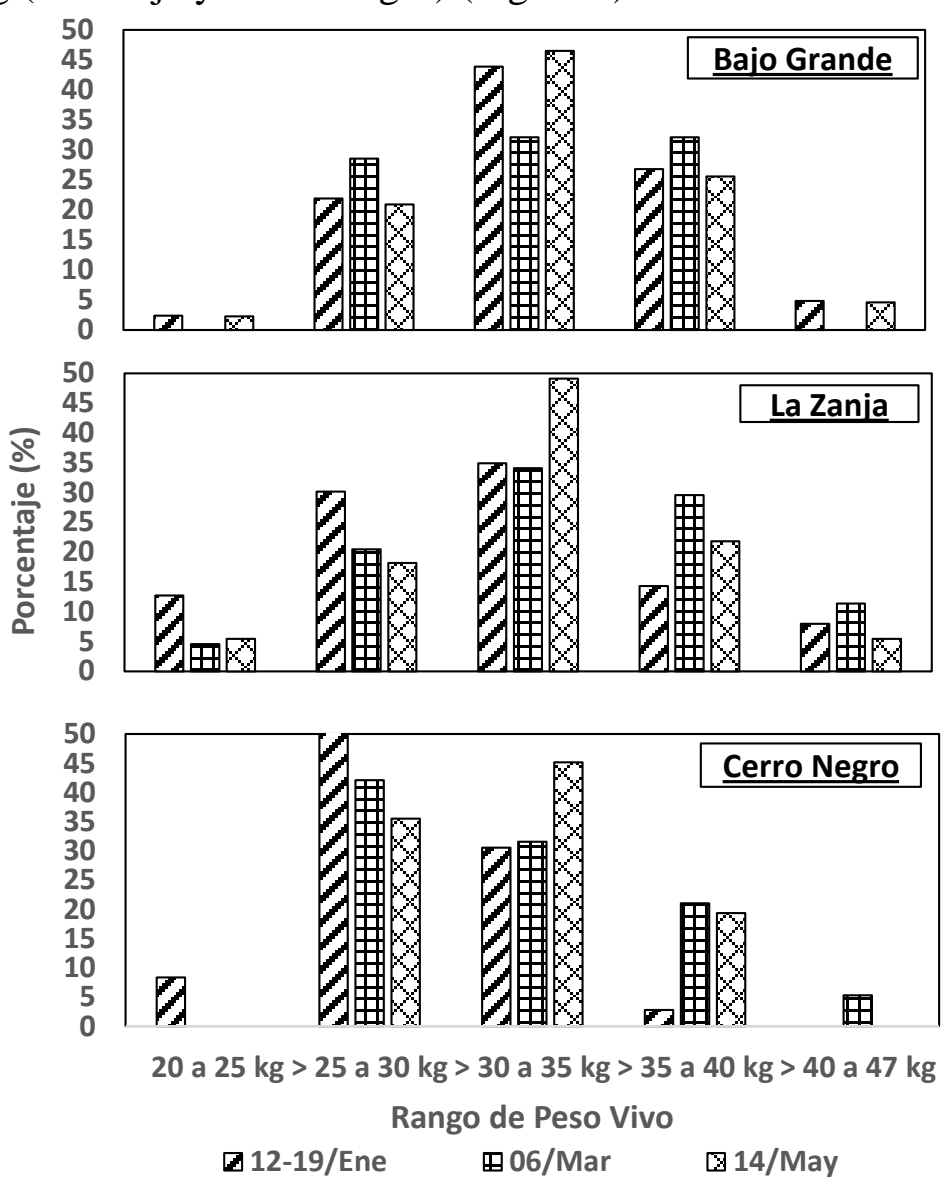

Figura 9: Distribución del porcentaje (\%) de corderas por rango de peso vivo y fecha para cada lote durante el uso del año 2017-2018. 
La evolución del PV de los lotes durante el $1^{\circ}$ año de uso de la pastura registró en los lotes La Zanja (LZ) y Argentino Abajo (AAB), respectivamente, pérdidas medias de 4,9 y 3,4 kg en el período 05-13/Ene al 16/Feb (34-42 días) debido al avanzado estado de madurez de la pastura, según Andrade et al. (2017). Asimismo, estos autores reportaron en 106-114 días de pastoreo pérdidas medias en los lotes descriptos de 3,3 y $2,9 \mathrm{~kg}$ que representaron reducciones del 11 $\%$ del PV inicial, y un predominio de los rangos de PV final de las corderas $>25$ a $30 \mathrm{~kg}$ y $>$ 20 a $25 \mathrm{~kg}$. En virtud de lo expuesto, la variación del PV de las corderas de estos lotes se relacionó $(\mathrm{p}<0,005)$ con los días transcurridos de uso de la pastura según modelos de regresión polinomial cuadrática (LZ: $y=30,03-0,190 x+0,001 x^{2}, R^{2}=0,20 ; A A B: y=25,78-$ $\left.0,127 \mathrm{x}+0,001 \mathrm{x}^{2}, \mathrm{R}^{2}=0,10\right)$. Además, el $\mathrm{PV}$ de los lotes descriptos estuvo correlacionado ( $\mathrm{p}<0,005)$, según Andrade et al. (2017), con el MV ( $\mathrm{r}=0,87$ y 0,94$), \mathrm{PB}(\mathrm{r}=0,84$ y 0,88$)$ y DIVM ( $\mathrm{r}=0,74$ y 0,77$)$ del forraje recolectado durante el uso de la pastura.

Por el contrario, en el período 12-17/Ene al 06/Mar (48-53 días) del $2^{\circ}$ año de uso de la pastura los lotes La Zanja (LZ) y Cerro Negro (CN) registraron ganancias de PV de 2,7 (0,051 $\mathrm{g} /$ día) y $3,1 \mathrm{~kg}(0,065 \mathrm{~g} / \mathrm{día})$, respectivamente, (Figuras 10 y $11 \mathrm{a}$ y b), que representaron aumentos del 10\% del PV inicial en ambos casos. Esta respuesta favorable podría deberse a un óptimo aprovechamiento de la pastura en la primera mitad del período informado en virtud del crecimiento de las plantas favorecido por la mayor pluviometría relevada en enero (Figura 1). Asimismo, los aumentos del PV de las corderas estuvieron reflejados en rangos de PV mayores el 06/Mar (> 30 a $35 \mathrm{~kg}$ y > 35 a $40 \mathrm{~kg}$ ) respecto al rango inicial predominante de PV (Figura 9). Cabe mencionar que, Utrilla et al. (2018) y Andrade et al. (2015) reportaron en corderas cruza aumentos del $25 \%$ (PVI y PVF: $22,0 \pm 4,1$ y $27,5 \pm 4,6 \mathrm{~kg}$ ) y $13 \%$ del PV (PVI y PVF: $25,3 \pm 4,5$ y $28,3 \pm 4,0 \mathrm{~kg}$ ) en los períodos diciembre-febrero y febrero-marzo, respectivamente, sobre la pastura de agropiros descripta de la EMS.

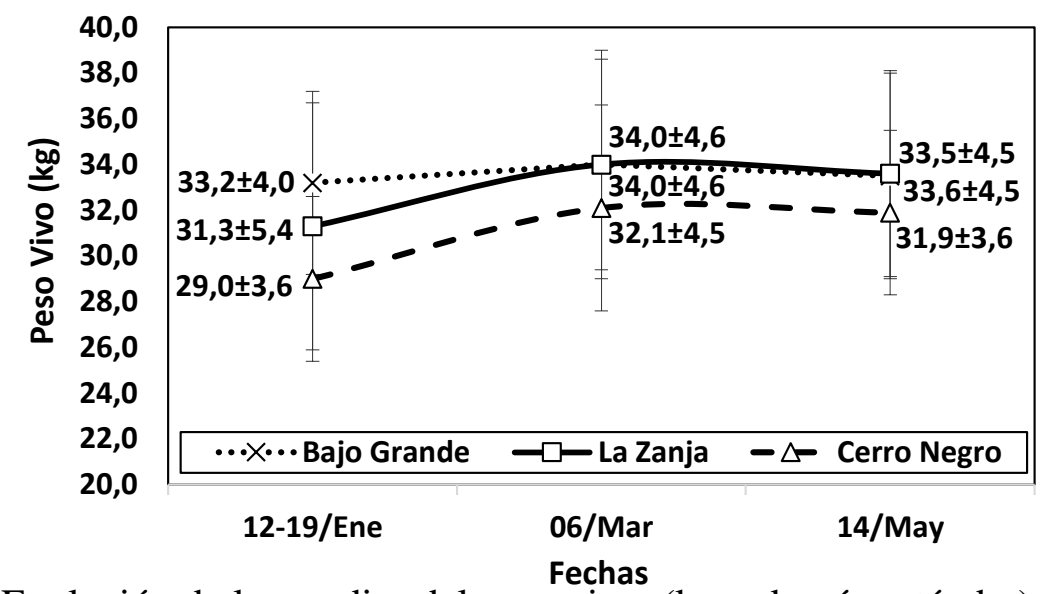

Figura 10: Evolución de las medias del peso vivo ( $\mathrm{kg} \pm$ desvío estándar) de las corderas por fecha y lote durante el uso del año 2017-2018. 

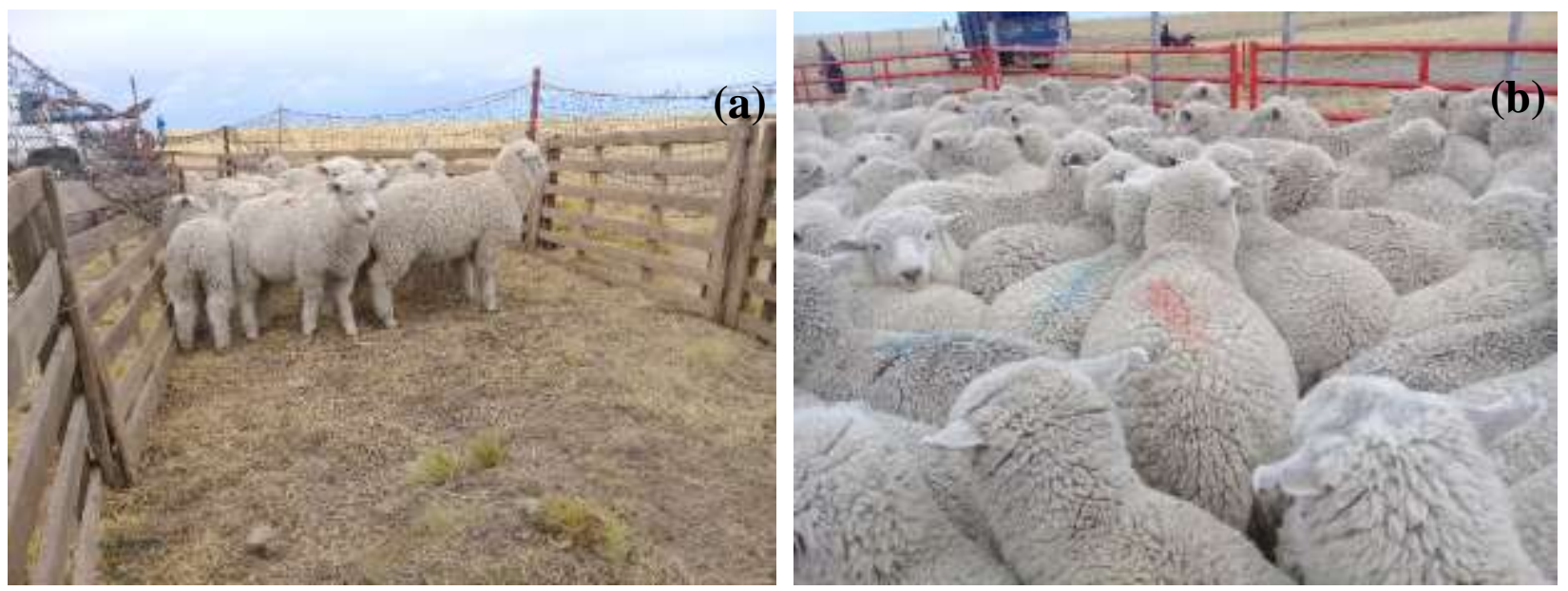

Figura 11: Vista frontal (a) y posterior de las corderas del lote La Zanja (b) previo al pesaje del 06/Mar.

En cambio, en el siguiente período (06/Mar-14/May) de 69 días se reportaron en los lotes descriptos pérdidas mínimas de PV (0,3-0,2 kg) (Figura 10), lo cual se vincularía principalmente con el avanzado estado de madurez del pasto ovillo (Figura 8a). A pesar de ello, al final del lapso de aprovechamiento de la pastura (117-122 días) las corderas provenientes de estos lotes manifestaron GPV de 2,4 (0,020 g/día) (LZ) y 2,9 kg (0,025 g/día) (CN) que representaron aumentos del 8 y $10 \%$ del PV. En concordancia con estos resultados, el rango predominante de $\mathrm{PV}$ en ambos lotes (> 30 a $35 \mathrm{~kg}$ ) representó una mejora respecto al rango de PV inicial más frecuente (Figura 9). En virtud de lo expuesto, la variación del PV de las corderas de estos lotes se relacionó $(\mathrm{p}<0,005)$ con los días de uso de la pastura según modelos de regresión polinomial cuadrática ( $\mathrm{LZ}: \mathrm{y}=31,21+0,084 \mathrm{x}-0,001 \mathrm{x}^{2}, \mathrm{R}^{2}=0,10 ; \mathrm{CN}: \mathrm{y}=$ $\left.28,87+0,092 x-0,001 x^{2}, R^{2}=0,13\right)$. En cambio, el PV de estos lotes se correlacionó ( $\left.<<0,005\right)$ negativamente con el MV $(r=-0,93$ y $r=-0,95)$ del forraje recolectado durante el aprovechamiento de la pastura.

Finalmente, el lote Bajo Grande no manifestó diferencias en la evolución del PV durante el lapso de uso de la pastura (Figura 10), lo cual estuvo reflejado en el mismo rango de PV inicial y final predominante relevado (> 30 a $35 \mathrm{~kg}$ ) (Figura 9). Cabe mencionar que, esta respuesta contrastante en relación a los lotes restantes podría explicarse por el gasto energético involucrado para mantener la mayor masa corporal de los animales del lote. Por ello, la variación del PV de las corderas no se relacionó ( $\mathrm{p}>0,05)$ con los días transcurridos según el modelo de regresión cuadrática evaluado.

\section{CONCLUSIONES}

La realización del presente trabajo permitió relevar la disminución del porcentaje de pérdidas de corderas a partir del manejo controlado en la pastura durante el período enero-mayo respecto al destete tradicional de los animales en el campo natural. En virtud de ello, esta práctica de manejo generó un beneficio global y con ello una mayor eficiencia en el sistema productivo en ejecución.

Asimismo, la experiencia pudo evaluar los cambios diferenciales del peso vivo (PV) de las corderas durante el lapso de aprovechamiento de la pastura en los años evaluados. En este contexto, fue determinante la pluviometría contrastante registrada el $1^{\circ}$ y $2^{\circ}$ año que influyo 
en la perfomance animal. Prueba de ello, fueron las pérdidas de PV de los animales durante el $1^{\circ}$ año, aunque manteniendo el rango de PV inicial y final, debido al estado madurativo precoz de la pastura por condiciones hídricas limitantes que afectaron su calidad nutricional. Al margen de ello, cabe mencionar el efecto negativo del estrés post-destete en la perfomance animal informado por Andrade et al. (2017). Por el contrario, la respuesta productiva general de las corderas durante el $2^{\circ}$ año fue una mejora en el PV y en el rango de PV final, en virtud de un óptimo aprovechamiento inicial de la pastura por mejores condiciones hídricas previas que favorecieron su crecimiento.

En este contexto, resulta necesario enfatizar la necesidad de optimizar el manejo de la pastura a partir de un pastoreo más temprano con animales jóvenes (borregas de reposición) que permita aprovechar en forma eficiente el mayor crecimiento primaveral del pasto ovillo establecido.

\section{AGRADECIMIENTOS}

Agradecemos la colaboración brindada por el personal de la Estancia Punta Loyola para el repunte, identificación y pesaje de los animales en las fechas establecidas y al Dr. Gabriel Oliva por la revisión del abstract del presente informe.

\section{BIBLIOGRAFÍA}

ANDRADE, M.; VARGAS, P.; CULUN, V. Y NUÑEZ, M. (2015). Resultados Preliminares del Aprovechamiento con animales de una Pastura de Agropiros Canadienses en el Campo Experimental Potrok Aike. (Informe Técnico de la EEA INTA Santa Cruz. 11p). Río Gallegos, Argentina. https://inta.gob.ar/sites/default/files/inta. https://doi.org/10.19137/semiarida.2017(01).19-25

ANDRADE, M.; UTRILlA, V.; VARGAS, P.; CLIFTON, G. Y ALVAREZ BENTO, J. (2017). Evaluación del aprovechamiento de una pastura de Pasto Ovillo y Agropiro Intermedio con corderas en Ea. Punta Loyola. (Informe Técnico de la EEA INTA Santa Cruz. 14 p). Río Gallegos, Argentina. https://inta.gob.ar/santacruz. https://doi.org/10.19137/semiarida.2017(01).19-25

AOAC INTERNATIONAL (formerly the Association of Official Analytical Chemists). (1995). Official Methods of Analysis. Arlington, VA: AOAC International. USA.

CORONATO, A.; MAZZONI, E.; VÁSQUEZ, M. Y CORONATO, F. (2017). Patagonia: Una síntesis de su Geografía Física. Capítulo Tercero: Clima. (pp. 57-69). Río Gallegos, Argentina. Ediciones Universidad Nacional de la Patagonia Austral. www.unpa.edu.ar/publicacion/version-digital-patagonia. https://doi.org/10.17227/01234870.41folios51.68

CLIFTON, G.; UTRILLA, V.; STURZENBAUM, M.V. Y RIVERA, E. (2012). Evaluación bajo corte y aprovechamiento de una pastura de agropiro pubescente (Agropyron trichophorum) con novillitos de engorde en el Sur de Santa Cruz. (En: Informe Final del Proyecto Regional (PATSU-910031): "Aumento de la productividad y competitividad de los sistemas bovinos en Patagonia Sur". 5 p). Río Gallegos, Argentina. Fuente: Acervo de la Biblioteca EEA INTA Santa Cruz. Contacto: lasagno.romina@inta.gob.ar. https://doi.org/10.4067/s0718-22442010000100016

DAUBENMIRE, R. (1959). A canopy coverage method for vegetational analysis. Northeast Science 33(1):43-64.

GALLI, J.R. (1997). Las pasturas como fuente de alimentación de rumiantes. En: C.A. Cangiano (Ed.). Producción animal en pastoreo. (pp. 27-40). EEA INTA Balcarce. Balcarce, Argentina. Ediciones INTA. 
HODGSON, J. (1990). Grazing management: Science into Practice. (203 p). Longman handbooks in agriculture. Longman Group UK Ltd.

MUNRO, J.M.M. Y WALTERS, R.J.K. (1985). The feeding value of grass. In: J. Frame (Ed.): Grazing. Occasional Symposium N ${ }^{\circ}$ 19, British Grassland Society. pp. 65-77.

OLIVA, G., GONZÁLEZ, L., RIAL, P. Y LIVRAGHI, E. (2001). Capítulo 2: El ambiente en la Patagonia Austral. En: P. Borrelli. y G. Oliva (Ed.), Ganadería Ovina Sustentable en la Patagonia Austral. (pp. 19-82). Centro Regional Patagonia Sur INTA. EEA INTA Santa Cruz. Río Gallegos, Argentina. Ediciones INTA. https://doi.org/10.4000/books.eunrn.616

PROYECTO REGIONAL TERRITORIAL: "Estrategias de intervención para el desarrollo agropecuario y agroalimentario en la zona Sur de Santa Cruz" (2013-2019) (PRET PATSU 129308. Centro Regional Patagonia Sur del INTA. EEA Santa Cruz. 35 p). Río Gallegos, Argentina. https://inta.gob.ar/santacruz/proyectos. https://doi.org/10.4067/s0718-22442016000100012

RIVERA, E.; STURZENBAUM, M.V.; UTRILLA, V.; MILICEVIC, F.; ROGEL B. Y CLIFTON, G. (2012). Evaluación del aprovechamiento y engorde de borregas sobre una pastura de agropiro intermedio (Agropyron intermedium). Ea. Las Horquetas. Santa Cruz. (Informe de Avance de la EEA INTA Santa Cruz. 6 p). Río Gallegos, Argentina. Fuente: Acervo de la Biblioteca EEA INTA Santa Cruz. Contacto: lasagno.romina@inta.gob.ar. https://doi.org/10.19137/semiarida.2017(02).2935

SAS. (2002-2008). User' Guide: Statistics. Version 9.2. SAS Institute Inc, Cary, NC, USA.

TILLEY J. M. A. Y TERRY R. A. (1963). A two-stage technique for the in vitro digestion of forage crops. J. Brit. Grassland Soc. 18:104-11. https://doi.org/10.1111/j.13652494.1963.tb00335.X

T'MANNETJE, L. Y HAYDOCK, K.P. (1963). The dry-weight-rank method for the botanical analysis of pasture. J.Brit. Grassland Soc. 18:268-275.

TOTHILL, J. C.; J. N. G. HARGREAVES; R. M. JONES Y MCDONALD, C.K. (1992). BOTANAL - A comprehensive sampling and computing procedure for estimating pasture yield and composition. 1. Field sampling. CSIRO Austr. Div. of Trop. Crops \& Past., Tropical Agronomy. Technical Memorandum N ${ }^{\circ} 78$.

UTRILLA, V.; STURZENBAUM, MARÍA V. Y RIVERA, E. (2007). "Evaluación de agropiros de origen canadiense y nacional en Ea. Punta Loyola”. (Informe Técnico de la E.E.A. Santa Cruz. AER Río Gallegos. 24 p). Río Gallegos, Argentina. Fuente: Acervo de la Biblioteca EEA INTA Santa Cruz. Contacto: lasagno.romina@inta.gob.ar. https://doi.org/10.4067/s0718-22442005000200008

UTRILLA, V.R.; STURZENBAUM, M.V. Y RIVERA, E.H. (2011). Evaluación de la producción y calidad forrajera de Agropiros en Santa Cruz. Rev.Arg.Prod.Anim. 31(I): 581.

UTRILLA, V.R.; ANDRADE, M.; VARGAS, P Y ALSINA, M.L. (2017). Relación del material vivo y muerto y calidad nutricional de una pastura de agropiros con factores físicos, ambientales y estado fenológico en la Patagonia Austral. Rev.Arg.Prod.Anim. 37 (I): 166.

UTRILLA, V.R.; VARGAS, P. Y ANDRADE, M. (2018). Desempeño productivo de corderas y ovejas y calidad nutricional de una pastura de agropiros en la Patagonia Austral. Rev.Arg.Prod.Anim. 38(I): 157

VARGAS, P.; ANDRADE, M. Y UTRILLA, V. (2016). Utilización de una pastura mezcla de agropiros con ovinos en el Sur de Santa Cruz. Comunicación. Rev.Arg.Prod.Anim. 36(I): 135 . 